\title{
Segmentation of Axonal Fibres in Tissue Slices
}

\author{
Nico Scherf ${ }^{1}$, Jens-Peer Kuska ${ }^{2}$, Claudia Heine ${ }^{1}$, Ulf-Dietrich Braumann ${ }^{1}$, \\ Heike Franke 3 \\ ${ }^{1}$ Translational Centre for Regenerative Medicine, University Leipzig, 04103 Leipzig \\ ${ }^{2}$ Interdisciplinary Centre for Bioinformatics, University Leipzig, 04107 Leipzig \\ ${ }^{3}$ Rudolf Boehm Institute of Pharmacology \& Toxicology, Univ. Leipzig, 04107 Leipzig \\ nscherf@trm.uni-leipzig.de
}

\begin{abstract}
This work focuses on the segmentation of axonal structures in digital images of organotypic slice co-cultures. An image processing chain is presented, which relies on anisotropic diffusion for preprocessing of the images and the intelligent scissors method for segmentation. This method requires manual user interaction to set the starting points. To overcome this drawback, the initial parameters for the intelligent scissors are automatically extracted from the images by a graph-based approach.
\end{abstract}

\section{Introduction}

The purpose of this work is to present an image processing chain for the segmentation of biocytin-traced axonal fibres in digital images of organotypic dopaminergic co-cultures of the rat brain. The segmentation step is crucial in order to be able to analyse the properties of axonal outgrowth depending on different factors (for the biological motivation behind this we refer the reader to [1]). A similar question was investigated by [2]. But whereas Pakura et al. were interested in the overall structure of the neural tissue, we like to analyse and compare the properties of single fibres. So we need a segmentation method able to track single fibres as exact as possible. The segmentation of line-like structures is a common problem in medical image processing (e.g. [3] or [4]). Most approaches base on global methods such as thresholding with additional local constraints depending on e.g. morphological features or curvature. We decided to use an anisotropic diffusion filter to enhance the line-like structures as well as to close small gaps along the fibres. The segmentation itself is obtained by the intelligent scissors approach [5] which leads to single spatially connected contours representing the axonal fibres. This method requires manual interaction. To overcome this disadvantage we propose a way to automatically extract the initial parameters for this segmentation from the image itself.

\section{Materials and Methods}

\subsection{Preprocessing}

The original images (an example is shown in Fig. 1(a)) are preprocessed to enhance the axonal structures for further segmentation. Since there is no useful 
colour information in the original images, we use a greyscale version instead. We are interested in the line-like structures, so at first a Laplacian of Gaussian (LoG) filter is applied to enhance the edges. This is shown in Fig. 1(b). The distribution of the grey-values along a single fibre is not homogeneous. This is due to the three-dimensional structure of the specimen under consideration (some parts of a fibre simply lie outside of the focal plane), and on the other hand the staining of the fibres itself might be inhomogeneous. We use a filter that enhances line-like structures and is able to close small gaps along a single axonal fibre. This is achieved by an anisotropic diffusion filter described in [6] that smoothes the image along the edges and therewith enhances the spatial coherence of the fibres. The resulting image is shown in Fig. 1(c). By this step, some structures of the background are also increased, so a further smoothing step is needed. Since we are interested in removing the background noise while preserving the important structures, a total-variation filter [7] is used. This filter only requires the standard deviation of the background noise as parameter. The TV-filtered image is depicted in Fig. 1(d).

\subsection{Segmentation}

As mentioned in the introduction, there is a great range of different segmentation strategies and none is superior to others in all possible contexts. When tracking the structure of a single fibre, on ideally wants a single spatially connected

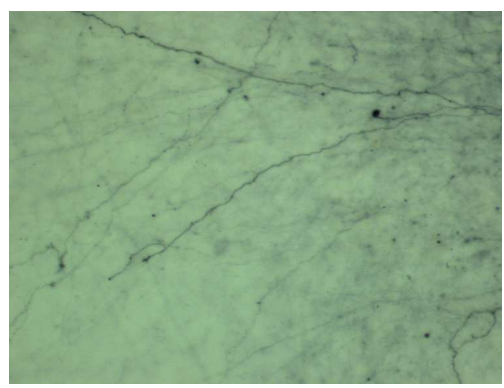

(a) Original image (specimen 1)

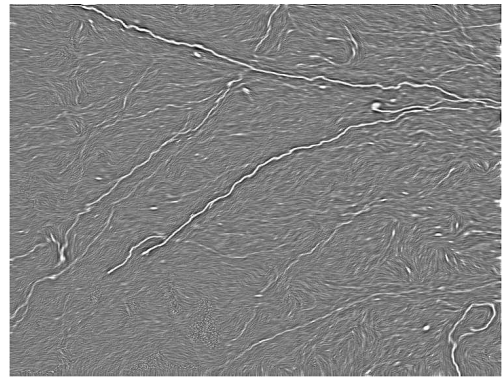

(c) Image after anisotropic diffusion

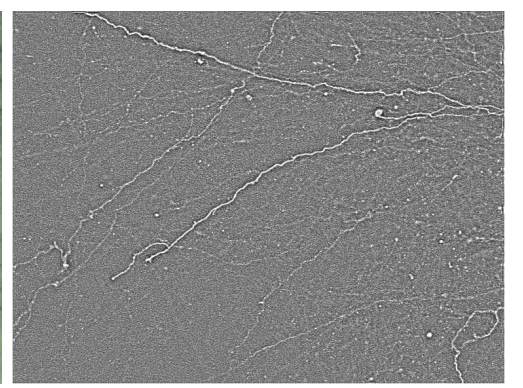

(b) Image filtered with LoG

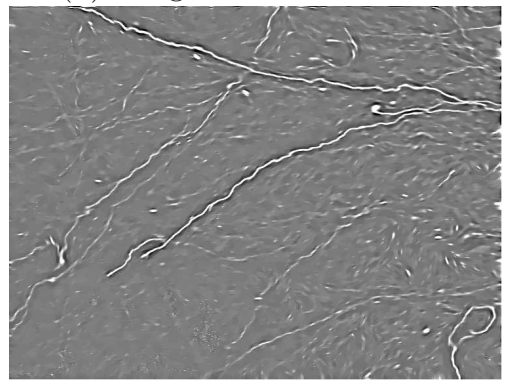

(d) TV-filtered image

Fig. 1. Preprocessing 
structure. When using morphological operators and thresholding, the resulting segmentation also consists of small structures that are not connected to a single fibre. In principle, this can be handled by removing structures that are too small (e.g. [2]). But this approach also removes small parts along the fibres, if there are between two gaps. An appropriate method for extracting the axons are the intelligent scissors proposed by [5]. By choosing two points on the fibre the intelligent scissors segmentation is able to track the path between them. The resulting contour is connected, so it is possible to interpolate gaps that occur. The segmentation result seems to be appropriate for analysing the properties of the axonal outgrowth, but this method has one great disadvantage: it is semiautomatic. User interaction is needed to place the initial points on the fibres. To overcome this problem, an automatic initialisation method, i.e. the extraction of reasonable starting points from the image itself is used.

\subsection{Parameter Extraction}

The quantification method presented by $[8]$ is able to extract a graph structure from images of astrocytes. Based on this approach we are able to extract points in the image, that lie on the ends of the fibres and therewith are ideal as initial points for the intelligent scissors segmentation. At first the preprocessed image is binarised by simple thresholding (Fig. 2(a)). No other thresholding method is used, since the grey-values in the filtered images nearly follow an ideal Gaussian distribution. From the binarised image, a graph structure of connected parts of the foreground is constructed (for details see [8]). Here, all structures with a number of vertices that is too small, are removed. The result is depicted in Fig. 2(b). Now the vertices of the graph (shown in red in Fig. 2(b)) are taken as initial points for the intelligent scissors. Two points are selected as initial pair, if their distance is above a given threshold. Then all combinations of such points are used as initialisation, leading to a bundle of possible fibre-tracks.

\section{Results}

An example of the results of the segmentation are shown in Fig. 3. One can see from this images, that the main fibre tracks can be segmented. A single fibre from the detected bundles is depicted in Fig. 3(b). The structure of the axonal outgrowth is well captured by the segmentation results. Fig. 3(c) and Fig. 3(d) show two further segmentation results from different specimens. One way of analysing the structure of a single extracted axon is to take the fibre as a one-dimensional signal and to look at the frequency distribution of this signal. As an example in Fig. 4 the structure of the fibre in Fig. 3(b) is plotted together with a log-log plot of the coefficients of the Fourier transform.

\section{Discussion}

While the segmentation method yields adequate results on the example images, extensive testing in laboratory practice will follow to demonstrate the method's 
Fig. 2. Feature Extraction

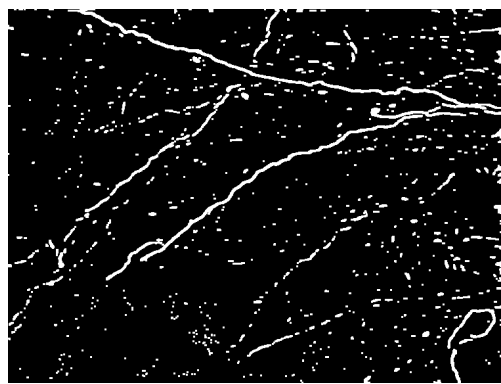

(a) Binarised image

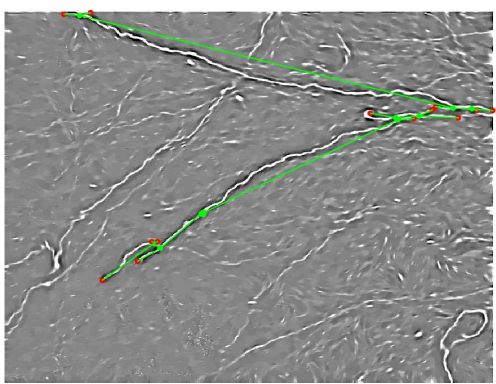

(b) Image graph plot

reliability. It is of great advantage that the resulting structures are connected lines, which can be used to analyse the different forms of growth of the axon fibres. One remaining point is the selection of single fibres from the bundles that are segmented. Since most combinations of start points lead to nearly the same structures (as can be seen from Fig. 3(a)) a simple way is to select the longest fibre from all the overlapping results as a representative. The aim of further work is the evaluation of other criteria for selecting a single representative fibre from the result as well as extracting additional features from the segmented fibres.

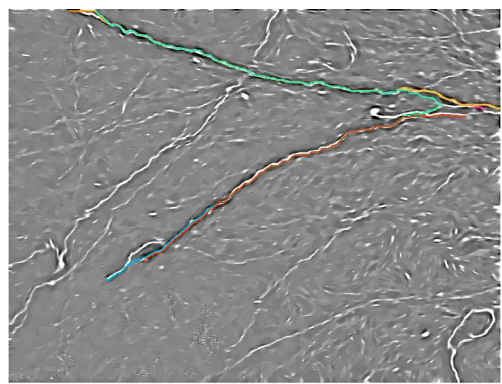

(a) All segmented fibres specimen 1

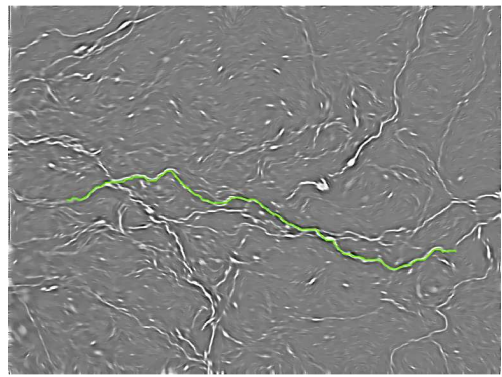

(c) Single fibre specimen 2

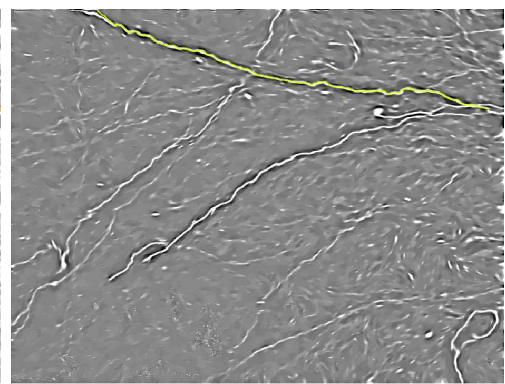

(b) Single fibre specimen 1

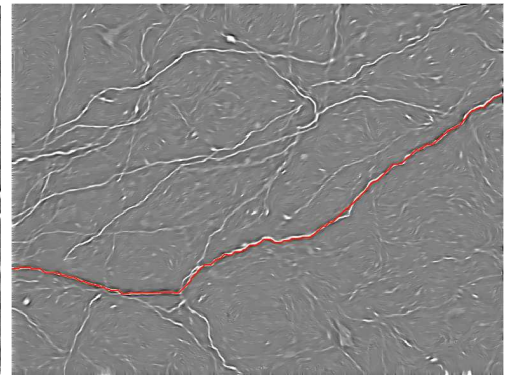

(d) Single fibre specimen 3

Fig. 3. Result of the intelligent scissors segmentation 
Fig. 4. Analysis of a single fibre

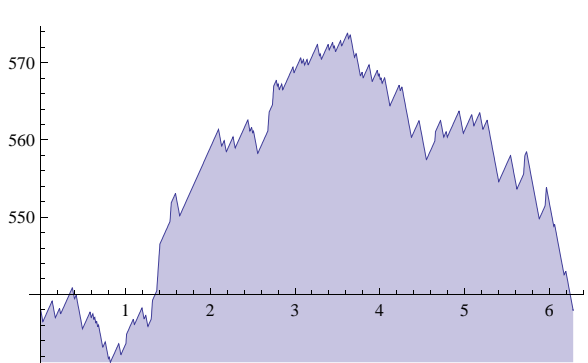

(a) Fibre as one-dimensional signal

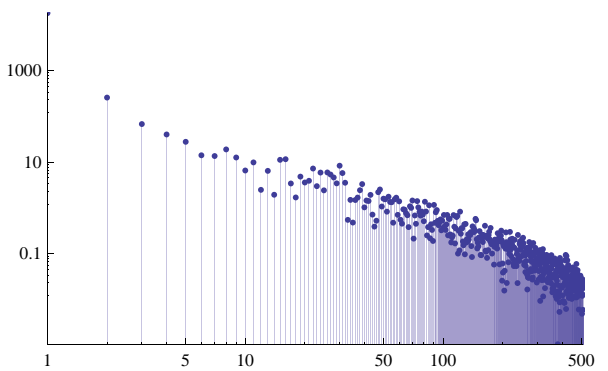

(b) Log-log plot of the Fourier coefficients

Acknowledgement. The project is supported by the Federal Ministry of Education and Research (BMBF), grant PTJ-BIO/31P4282 (MS CartPro) and grant PTJ-BIO/031390 (TRM Leipzig).

\section{References}

1. Heine C, Wegner A, Grosche J, et al. P2 receptor expression in the dopaminergic system of the rat brain during development. Neuroscience. 2007;149(1):165-81.

2. Pakura M, Schmitt O, Aach T. Segmentation and analysis of nerve fibers in histologic sections of the cerebral human cortex. Proc SSIAI. 2002; p. 62-6.

3. Zana F, Klein JC. Segmentation of vessel-like patterns using mathematical morphology and curvature evaluation. IEEE Trans Image Process. 2001;10(7):1010-9.

4. Jelinek HF, Cree MJ, Leandroa JJG, et al. Automated segmentation of retinal blood vessels and identification of proliferative diabetic retinopathy. J Opt Soc Am A. $2007 ; 24(5): 1448-56$.

5. Mortensen EN, Barrett WA. Intelligent scissors for image composition. Proc SIGGRAPH. 1995; p. 191-8.

6. Weickert J. Anisotropic Diffusion in Image Processing. Teubner; 1998.

7. Chan TF, Osher S, Shen J. The digital TV filter and nonlinear denoising. IEEE Trans Image Process. 2001;10(2):231-41.

8. Braumann UD, Franke H, Hengstler J, et al. Graph-based quantification of astrocytes. Proc BVM. 2006; p. 379-83. 